\title{
Aperture masking behind AO systems
}

\author{
Michael J. Ireland ${ }^{a, b, c}$ \\ ${ }^{a}$ Department of Physics and Astronomy, Macquarie University, NSW 2109, Australia; \\ ${ }^{b}$ Australian Astronomical Observatory, PO Box 296, Epping, NSW 2121, Australia; \\ ${ }^{c} \mathrm{MQ}$ Research Centre in Astronomy, Astrophysics and Astrophotonics, Macquarie University, \\ NSW 2109, Australia
}

\begin{abstract}
Sparse Aperture-Mask Interferometry (SAM or NRM) behind Adaptive Optics (AO) has now come of age, with more than a dozen astronomy papers published from several $5-10 \mathrm{~m}$ class telescopes around the world. I will describe the reasons behind its success in achieving relatively high contrasts ( 1000:1 at lambda/D) and repeatable binary astronomy at the diffraction limit, even when used behind laser-guide star adaptive optics. Placed within the context of AO calibration, the information in an image can be split into pupil-plane phase, Fourier amplitude and closure-phase. It is the closure-phase observable, or its generalisation to Kernel phase, that is immune to pupil-plane phase errors at first and second-order and has been the reason for the technique's success. I will outline the limitations of the technique and the prospects for aperture-masking and related techniques in the future.
\end{abstract}

Keywords: aperture mask interferometry, sparse aperture masking, extrasolar planets, adaptive optics, optical interferometry

\section{INTRODUCTION}

The calibration of Adaptics Optics (AO) images has long been a key limitation in achieving the full diffraction limit of large telescopes. The photon-noise limit for diffraction-limited imaging gives $>10$ magnitudes contrast at 5- $\sigma$ for 10 minute exposures on $\mathrm{K} \sim 10$ magnitude stars where a companion is buried in the airy-ring of a $10 \mathrm{~m}$ class telescope Point-Spread Function (PSF). However, PSFs have not been able to be calibrated nearly this well, meaning that imaging at the full diffraction limit has been restricted to moderate contrasts ( $<4$ magnitudes): the regime of binary star science. Even in the case of binary stars, precision astrometry has been difficult, because this also requires precise PSF calibration.

Sparse Aperture-Mask interferometry is a technique borrowed from calibration of seeing-limited observations $^{1,2}$ that enables a more precise calibration of the PSF at the diffraction-limit. It has been used since 2004 behind adaptive optics systems, mostly to enable high-contrast diffraction-limited imaging and precise binary astrometry. ${ }^{3-5}$ This technique involves placing a binary mask in the pupil-plane of the telescope, where the majority of the mask is opaque. These masks as commissioned have consisted of arrays of holes where the vector separation between any two holes is unique. The terms Non-Redundant Masking (NRM) or Sparse Aperture Masking (SAM) have both been used, partly reflecting origins in US publications (NRM) or European publications (SAM). As will be seen in Section 2, SAM is technically more correct because all holes of non-zero size are redundant, but NRM is possibly a more descriptive term.

In this paper, I will review SAM as it applies behind AO systems, attempting to repeat little of what has come before, and also attempting to minimise overlap with other papers presented at this conference. ${ }^{6,7} \mathrm{I}$ will give a background to SAM in section 2, then I will discuss the general problem of phase errors in AO imaging in section 3. I will discuss the calibration of kernel/closure phase in section 5 , and how these calibrated observables can be used for high-contrast imaging and astrometry in sections 6 and 7. Finally, I will conclude and discuss the future of SAM in the context of the next generation of telescopes and AO systems in section 8.

\footnotetext{
E-mail: michael.ireland@mq.edu.au
} 

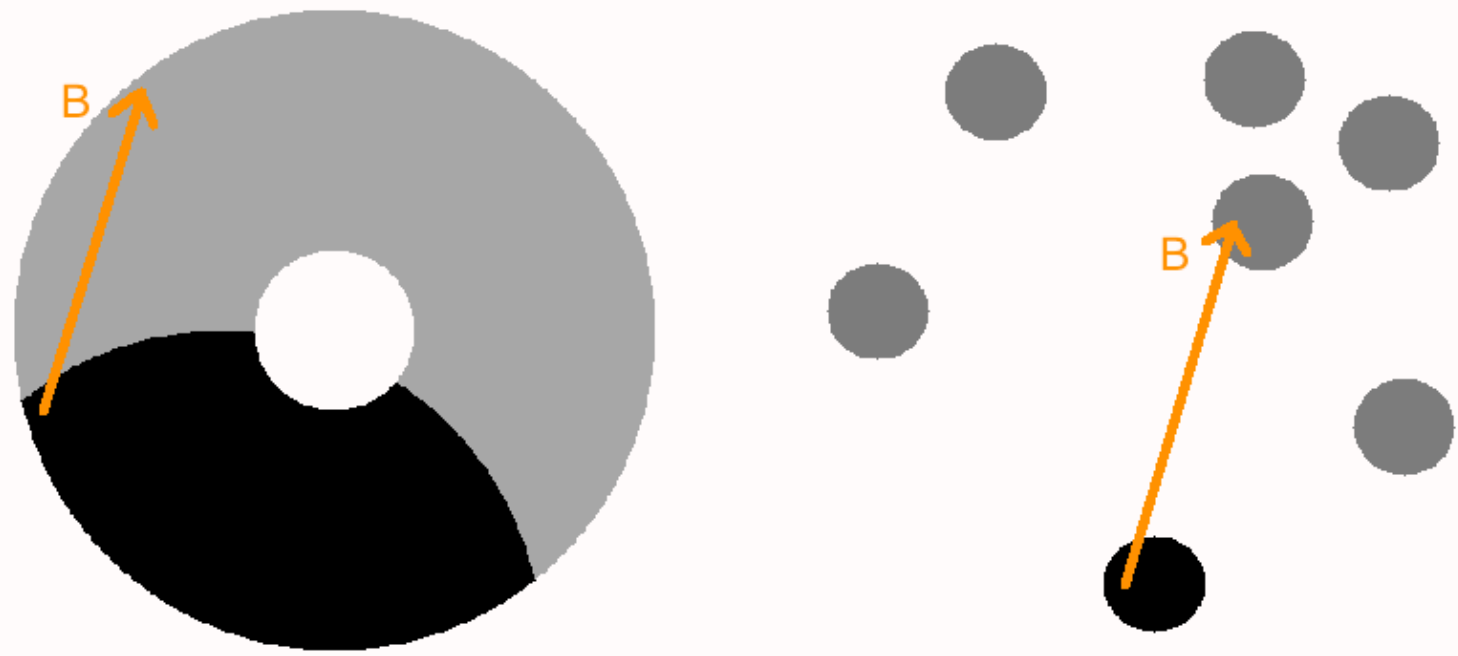

Figure 1. An illustration of the support function $S$ (in black) for a baseline vector $B=(u, v)$ on a typical circular pupil with obstruction (left) and a sparse aperture mask (right). The sparse aperture is clearly less sensitive to large scale phase errors.

\section{SPARSE APERTURE MASKING BACKGROUND}

Sparse aperture-masking is a form of high-resolution imaging where the amplitude and phase of the Fourier transform of the image are considered the primary observables, and the information contained in the Fourier domain is as carefully controlled as possible. Consider images formed by a complex pupil-plane aperture function $P(x, y)$. The Fourier-transform of the image formed by this pupil is the auto-correlation of the aperture-function:

$$
F(u, v)=\int P(x+u, y+v) P^{*}(x, y) d x d y
$$

For this to make sense dimensionally, if $x$ and $y$ have units of meters, $u$ and $v$ also have units of meters, and spatial frequency is converted to cycles per radian by dividing by wavelength. For a simple aperture function $P$ that is simply zero where light is blocked and $\exp (i \phi(x, y))$ for some aberration function $\phi$, we can simplify this integral to:

$$
F(u, v)=\int \exp (i[\phi(x+u, y+v)-\phi(x, y)]) S(x, y) d x d y,
$$

where the support function $S$ is 1 where baselines exist within the pupil, and 0 elsewhere (see Figure 1 and 2).

Historically, mask holes have were made even smaller than in Figure 1, in order to match the coherence length in speckle imaging. ${ }^{2}$ The structure function in speckle imaging was roughly Kolmogorov, meaning that the majority of the phase aberrations was on large spatial scales. In this case, given sufficient flux, a nonredundant aperture-mask meant that there phase was nearly constant (and certainly didn't wrap) in the integral of Equation 2, producing high fringe contrast. This provided a clear advantage over full pupil imaging. The problem with speckle imaging through an aperture-mask was that there were very few science targets for which sufficient signal-to-noise could be obtained within an atmospheric coherence time. SAM with AO has pushed to fainter magnitude limits, meaning larger sub-aperture diameters. The combination of larger sub-aperture diameters with AO correction of the large spatial scale aberrations means that the advantages of SAM are no longer so clear, and have to be examined in detail. 

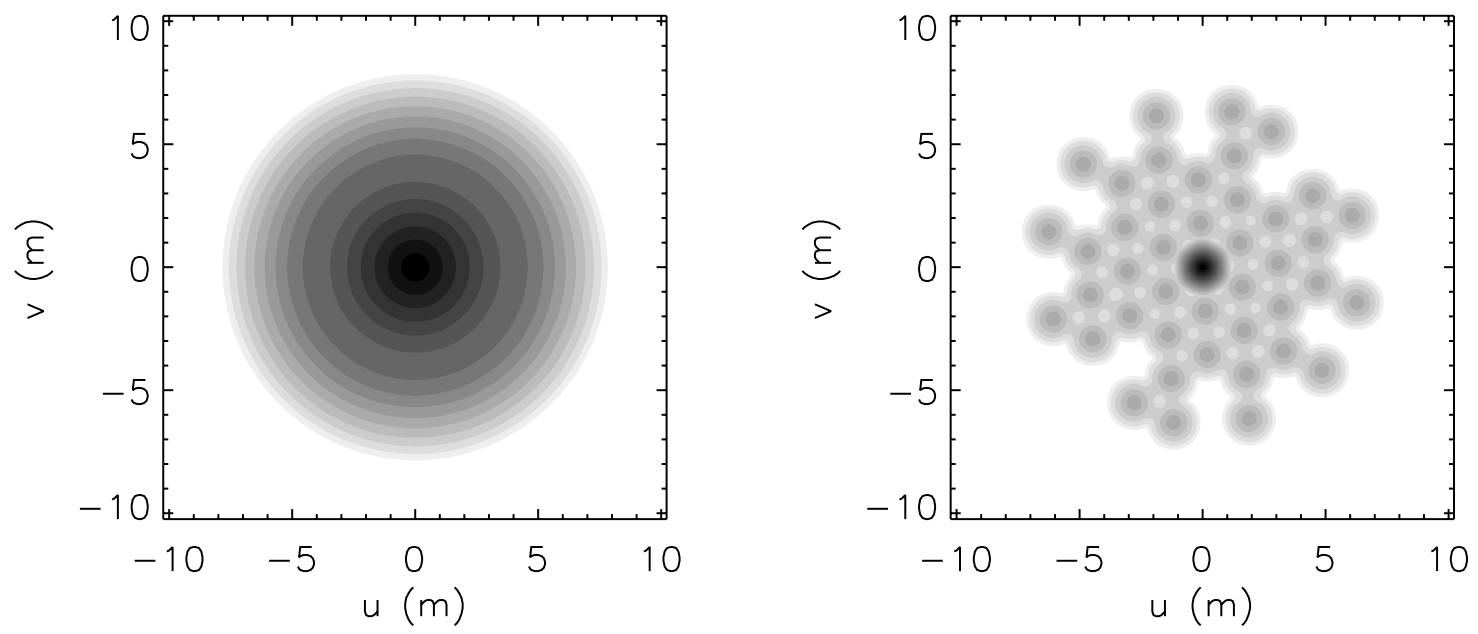

Figure 2. The autocorrelations of the aperture functions illustrated in Figure 1.

\section{PHASE ERRORS IN AO IMAGING}

Fourier amplitudes are roughly proportional to Strehl ratio, so change with changing Strehl and are difficult to calibrate. They are discussed briefly in the interferometry session of this conference. ${ }^{6}$ In this paper, we will consider Fourier phase only. We can consider phase in Equation 2 more carefully by approximating the argument of $F$ to first order in $\phi$ :

$$
\begin{aligned}
F(u, v) & =\int S d x d y+i \int \Delta_{u, v} \phi S d x d y, \text { where } \\
\Delta_{u, v} \phi(x, y) & =\phi(x+u, y+v)-\phi(x, y) \\
\operatorname{Arg}(F(u, v)) & =\left(\int \Delta_{u, v} \phi S d x d y\right) /\left(\int S d x d y\right)+O\left(\phi^{3}\right) .
\end{aligned}
$$

In the case of a finite field of view, the image Fourier transform $F$ becomes discrete. We can then replace Equation 5 with a sum:

$$
\psi_{k}=\Sigma_{j=1}^{N_{P}} a_{k, j} \phi_{j}
$$

Here $\psi_{k}$ is a Fourier phase, meaning an argument of $F(u, v)$ for a discrete Fourier pixel $\left(u_{k}, v_{k}\right)$. $\phi_{j}$ is similarly a pupil-plane phase for pupil location $\left(x_{j}, y_{j}\right)$. For a 2-dimensional aperture, there are always at least twice as many elements $\psi_{k}$ as there are elements $\phi_{j}$. To see why, consider the most redundant aperture, a fully filled shape, in this case a circle. For an aperture with diameter $D$ pixels that includes the longest baselines in the pupil (the aperture edge), there are $\pi D^{2} / 4$ free pupil-plane phases, and $\pi D^{2} / 2$ measured Fourier phases. The factor of 2 in Fourier plane phases is due to the complex Fourier amplitude at $(-u,-v)$ being the complex conjugate of a Fourier amplitude at $(u, v)$. An annular aperture, or any other sparse aperture, has the same number of Fourier phases but fewer pupil phases.

This means that, to first order, at least half the phase information measured in the Fourier transform of an image is independent of phase aberrations. This extremely powerful piece of information has not been explicitly used in AO imaging, however it is beginning to be now following on from the concept of kernel phase outlined by Martinache (2010). ${ }^{8}$ The technique is conceptually simple - the range of the matrix $A=\left\{a_{k, j}\right\}$ forms a subspace $R$ in the Fourier-phase vector space $V=\left\{\psi_{1}, \phi_{2}, \ldots, \psi_{N_{F}}\right\}$. The orthogonal complement of $R$ in $V$ is the set 

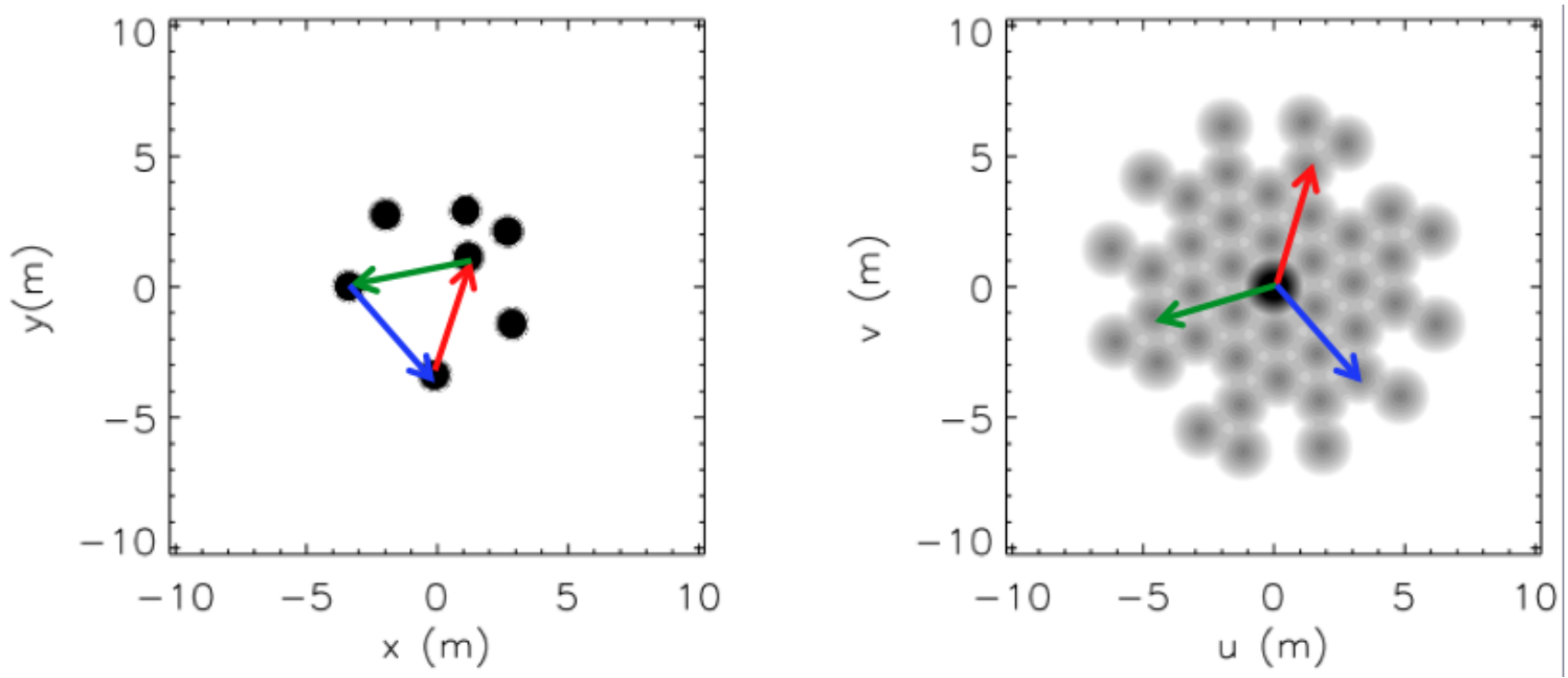

Figure 3. Left: The same pupil as in the right hand panel of Figure 1, with a closing triangle highlighted. Right: The locations of the Fourier phases corresponding to this closing triangle. A closure-phase is formed by the sum of the Fourier phase at these locations.

of kernel-phases $K$, consisting of linear combinations of Fourier phases that are independent to first order of pupil-plane phases $\phi_{j}$. By projecting the Fourier phases $\psi_{k}$ onto the subspace $K$ using standard linear algebra techniques, we arrive at a set of $N_{F}-N_{P}+1$ kernel phases (the " +1 " comes from piston ${ }^{8}$ ).

Kernel-phase is closely related to techniques based on bispectrum phase ${ }^{9}$ and indeed any paper that fits to bispectrum phase ${ }^{10}$ is essentially fitting to kernel-phase. Although each bispectrum-phase is a kernel-phase, there are many more bispectrum phases than kernel-phases, meaning that it is difficult to be statistically robust when fitting to bispectrum phase. In this context, kernel-phases can be thought of as linearly independent linear combinations of bispectrum phases. The advantage of the bispectrum is that it can be used when phase itself is not a robust observable - i.e. when there is a significant probability in each frame of data that a Fourier amplitude is zero within errors. In this case, the complex bispectrum is averaged. The advantage of kernel-phase over bispectrum phase is that the problem of computing an using the covariance matrix is tractable (to be described in Ireland 2012, in prep).

\section{CLOSURE-PHASE AND LOW-STREHL DATA}

Rather than attempting a full kernel-phase analysis, SAM data has typically been analysed by approximating the phase across each sub-aperture as being constant. With $n$ subapertures, this gives $n(n-1) / 2$ discrete Fourier phases, $(n-1)(n-2) / 2$ Kernel-phases, and $n(n-1)(n-2) / 6$ closure-phases. The concept of a closure-phase is illustrated in Figure 3 for the example of the 7-hole mask. Sampling the Fourier plane in 3 locations and adding up the phases gives a closure-phase, which is just one example of a kernel-phase. Just like in bispectrum speckle interferometry, rather than computing phase directly for single exposures, we can compute closure-phase by computing the triple-product corresponding to each closure-phase. Averaging the triple product is a convenient way to give a low weight to images with low Fourier amplitudes, and also to solve the phase wrapping problem. The argument of the triple product is the closure-phase. For modern telescopes, this is only particularly useful for low-Strehl data using the CONICA camera, where a fast acquisition mode called a cube mode is available.

Even where phase is a good observable in single exposures, there is an advantage to using an aperture-mask over imaging with a fully-illuminated pupil. This is clear from Figure 1: if there are dominant large-scale aberrations, the third-order phase errors in Equation 5 will contribute much more to the kernel-phase for the fully-illuminated pupil when compared to the sparse aperture mask. This advantage of a sparse aperture-mask is especially useful for low-Strehl data typical of a laser-guide star system. Defocus in particular can be large if 
the slow wavefront sensor is not able to adequately track the changing height of the sodium layer. The ability of aperture-masking to enable diffraction-limited resolution for this kind of data has been used sucessfully in the studies of brown dwarf binaries[CITATION].

\section{PHASE CALIBRATION}

In principle, the kernel- and closure- phase techniques do not require calibration, in a similar manner to raw adaptive optics images not requiring calibration. In the case of laser-guide star observations, calibrator stars are typically not used, because of the overheads associated with taking data on a calibrator, the relatively noisy data and contrast requirements that are relatively easily met. However, for most SAM data, the systematic closure-phases caused by third-order pupil-plane phase errors need to be calibrated. The standard method of calibration is inspired by speckle and long-baseline interferometry, where a single unresolved calibrator star is observed, so that the closure- or kernel-phases from this star can be subtracted from the target. Early SAM observations ensured that the AO WFS magnitude and the infrared magnitude was similar between target and calibrator. This requirement has since been relaxed - as long as the AO WFS has a good background (i.e. so that there isn't a brightness-dependent static aberration), calibration benefits from higher Strehls and higher photon-limited signal-to-noise.

An ideal calibrator is one that is co-located with the target and observed simultaneously. As this is not possible, many calibrators can be observed, and an optimal linear combination of calibrators used to minimise the closure- or kernel- phase residuals. These techniques are described in other references ${ }^{6,11}$ and will be expanded in an upcoming publication (Ireland 2012, in prep).

\section{HIGH-CONTRAST IMAGING}

So far, we have discussed kernel- and closure- phases as arbitrary observables. In order to determine the structure of the target, we need to be able to fit to the data. The forward model is simple to compute for kernel- and closure-phase - a Fourier transform is taken of a model image and the kernel- or closure-phase computed, and a $\chi^{2}$ value computed. Code such as MACIM $^{12}$ can then minimise $\chi^{2}$ and find the best fitting image. However, not all images give a signal in Fourier-phase. In particular, any point-symmetric target has only 2 possible values of Fourier-phase: 0 or 180 degrees. In general, creating images from phase data alone is a difficult process, but in the high-contrast regime, where there is an unresolved source containing the majority of the flux, the situation is simplified. A model image $I$ can be decomposed into its point-symmetric and point-antisymmetric components:

$$
\begin{gathered}
I_{s}=\left(I_{0}+I_{180}\right) / 2 \\
I_{a}=\left(I_{0}-I_{180}\right) / 2 .
\end{gathered}
$$

Here $I_{a}$ is the antisymmetric image, and $I_{s}$ is the symmetric image. $I_{180}$ is the image rotated by 180 degrees, and $I_{0}$ is the true image. Fitting to phase alone gives $I_{a}$ only. As this has negative flux, there is a minimum possible $I_{a}$ to produce image positivity. However, with phase data alone, adding more than this minimum $I_{a}$ is possible without modifying the fit.

In practice, imaging from phase data needs care, and model-fitting for simple targets (such as a star and one or more faint companions) is preferred. The contrast limit to high-contrast imaging using aperture-masking has several causes:

- Photon- or background-noise. This is typically only a dominant error for LGS data (K magnitudes around 12 or fainter) or for L-band or longer wavelengths. Locking an adaptive optics loop requires or order $10^{4}$ photons per second per sub-aperture. The contrast limit is approximately the square-root of the number of photons collected per sub-aperture. If we approximate the number of infrared photons through a broadband filter to be the same as the number of $\mathrm{AO}$ wavefront sensor photons, then a signal-to-noise of $10^{3}$ is obtained in only $100 \mathrm{~s}$ whenever the AO loops are locked. 
- Sub-aperture phase noise. Equation 5 is only valid to first-order, with residuals that are third-order in pupil-plane phase $\phi$ over the sub-aperture. This causes both an unbiased closure-phase error, and a closurephase bias whenever aberrations differ between a target and a calibrator. Carefully calibrated data has demonstrated approximately 6 magnitudes of contrast in 15 minutes of on-sky time at 5- $\sigma$, with contrast improving as square-root time.

- Dispersion-related phase noise. For broad-band filters, the combination of atmospheric dispersion and differing airmasses or spectra cause closure-phase systematics. This effect can be largely removed by careful calibration, or choosing narrower bandwidth filters (or an IFU).

- Subaperture piston noise. Given that the exposure times used in SAM are typically much longer than the atmospheric coherence time, time-variable piston causes non-zero closure phases . For bright, wellcalibrated data, the contrast limits to SAM (about 1000:1) are generally consistent with simulations that include these effects.

\section{PRECISION ASTROMETRY}

Given that fitting to closure- or kernel-phase is unbiased (i.e. calibration errors are equally likely to give positive or negative phases) and ensemble averages give well defined error bars, SAM is a fantastic technique for computing precision orbits of binary stars. An example of this precision is shown in Figure 4, with more detail given in several refereed papers. ${ }^{10,13,14}$ When astrometric targets are observed in a group with interspersed calibrators, approximately 8 minutes of observing time at Keck is required for one astrometric data point, including all overheads.

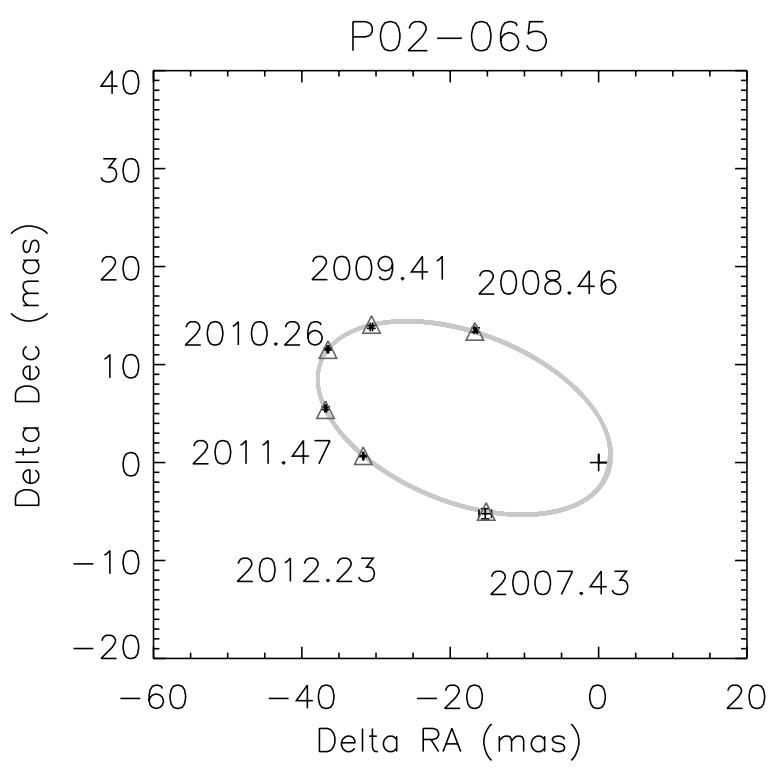

Figure 4. An orbit for the young binary star [PBB2002] 160517.9-202420. Typical residuals are 100 $\mu$ as, with a combination of K-band and CH4S (1.55 micron filter) data from Keck plotted. The smallest separation measured is 16 mas in Kband, corresponding to $0.35 \lambda / \mathrm{D}$. Contrast is $\sim 0.4$ magnitudes (thanks to A. Kraus for access to the data in advance of publication).

\section{CONCLUSIONS AND FUTURE WORK}

Sparse Aperture Masking (SAM) has been a successful technique to achieve the full diffraction-limit of large telescopes. Precisely calibrated data can be obtained through the kernel-phase observable, which includes closurephase observables as a subset. The contrast obtained by this technique is approaching the limits imposed by the performance of adaptive optics systems and the photon-noise limit (around 7 magnitudes for 1 our on sky). The 
use of SAM with IFUs promises to both remove dispersion-related phase noise, and give spectral properties of targets with a single observation. SAM will continue to be the preferred technique for binary astrometry at the diffraction limit of the current and next generation of large telescopes, especially for moderate Strehl ratio data expected to be typical of LGS AO systems.

\section{ACKNOWLEDGMENTS}

Although this invited paper lists only one author, this paper builds on the previous work, discussions with and encouragement of a large number of people. In particular, I would like to thank Tom Evans, Adam Kraus, Shri Kulkarni, Sylvestre Lacour, David Lafreniére, James Lloyd, Frantz Martinache, John Monnier, Laurent Puyeyo, Anand Sivaramakrishnan and Peter Tuthill.

\section{REFERENCES}

1. Baldwin, J. E., Haniff, C. A., Mackay, C. D., and Warner, P. J., "Closure phase in high-resolution optical imaging," Nature 320, 595-597 (Apr. 1986).

2. Tuthill, P. G., Monnier, J. D., Danchi, W. C., Wishnow, E. H., and Haniff, C. A., "Michelson Interferometry with the Keck I Telescope," PASP 112, 555 (2000).

3. Lloyd, J. P., Martinache, F., Ireland, M. J., Monnier, J. D., Pravdo, S. H., Shaklan, S. B., and Tuthill, P. G., "Direct Detection of the Brown Dwarf GJ 802B with Adaptive Optics Masking Interferometry," ApJ 650, L131-L134 (Oct. 2006).

4. Tuthill, P., Lloyd, J., Ireland, M., Martinache, F., Monnier, J., Woodruff, H., ten Brummelaar, T., Turner, N., and Townes, C., "Sparse-aperture adaptive optics," Proc. SPIE Vol. 6272, p.62723A, Advances in Adaptive Optics II, Ellerbroek, Calia; Ed. 6272 (July 2006).

5. Lacour, S., Tuthill, P., Amico, P., Ireland, M., Ehrenreich, D., Huelamo, N., and Lagrange, A.-M., "Sparse aperture masking at the VLT. I. Faint companion detection limits for the two debris disk stars HD 92945 and HD 141569," A\&A 532, A72 (Aug. 2011).

6. Ireland, M., "Detecting Extrasolar Planets with sparse Aperture Masking," Proc. SPIE Vol. 8445, Optical and Infrared Interferometry III 8445, 5 (2012).

7. Norris, B., Tuthill, P., Ireland, M., Lacour, S., Zijlstra, A., Lykou, F., Evans, T., Stewart, P., Bedding, T., Guyon, O., and Martinache, F., "Probing dusty circumstellar environments with polarimetric aperturemasking interferometry.," Proc. SPIE Vol. 8445, Optical and Infrared Interferometry III 8445, 2 (2012).

8. Martinache, F., "Kernel Phase in Fizeau Interferometry," ApJ 724, 464-469 (Nov. 2010).

9. Lohmann, A. W., Weigelt, G., and Wirnitzer, B., "Speckle masking in astronomy - Triple correlation theory and applications," Appl. Opt. 22, 4028-4037 (Dec. 1983).

10. Ireland, M. J., Kraus, A., Martinache, F., Lloyd, J. P., and Tuthill, P. G., "Dynamical Mass of GJ 802B: A Brown Dwarf in a Triple System," ApJ 678, 463-471 (May 2008).

11. Kraus, A. L. and Ireland, M. J., "LkCa 15: A Young Exoplanet Caught at Formation?," ApJ 745, 5 (Jan. 2012).

12. Ireland, M. J., Monnier, J. D., and Thureau, N., "Monte-Carlo imaging for optical interferometry," Advances in Stellar Interferometry. Edited by Monnier, John D.; Schöller, Markus; Danchi, William C.. Proceedings of the SPIE, Volume 6268, pp. (2006). (July 2006).

13. Dupuy, T. J., Liu, M. C., and Ireland, M. J., "Keck Laser Guide Star Adaptive Optics Monitoring of the M8+L7 Binary LHS 2397aAB: First Dynamical Mass Benchmark at the L/T Transition," ApJ 699, 168-185 (July 2009).

14. Evans, T. M., Ireland, M. J., Kraus, A. L., Martinache, F., Stewart, P., Tuthill, P. G., Lacour, S., Carpenter, J. M., and Hillenbrand, L. A., "Mapping the Shores of the Brown Dwarf Desert. III. Young Moving Groups," ApJ 744, 120 (Jan. 2012). 